\title{
Small Bowel Gastrointestinal Stromal Tumor as a Gateway for Streptococcus anginosus Causing Multiple Liver Abscesses
}

\author{
Gabriella A. Conte ${ }^{\mathrm{a}, \mathrm{f}}$, Jonathan S. Harmon ${ }^{\mathrm{b}}$, Rachel A. Masia ${ }^{\mathrm{c}}$, Diane Marchesani ${ }^{\mathrm{a}}$, \\ Xiu Sun ${ }^{\mathrm{d}}$, Elsa Marisol Pichardo ${ }^{\mathrm{c}}$, Fernando Brea Parrilla ${ }^{\mathrm{e}}$, \\ Michael J. Levitt ${ }^{\mathrm{a}}$, Angelo A. Chinnici ${ }^{\mathrm{a}}$
}

\begin{abstract}
Gastrointestinal stromal tumors (GISTs) are the most common type of mesenchymal neoplasm of the gastrointestinal tract but consist of only $1 \%$ of all primary gastrointestinal neoplasms. Differentiated from other spindle cell tumors, GISTs are uniquely positive for CD117 expression which allows for molecular targeting therapy with imatinib mesylate (Gleevec). Clinical presentations are variable, ranging from asymptomatic to vague symptoms of abdominal pain, early satiety, abdominal distention or gastrointestinal bleeding. Very rarely, patients can present with tumor-bowel fistula and intra-abdominal abscesses. In this article, we discuss a rare presentation of a middle-aged male with multiple liver abscesses found to have a primary small bowel GIST. This patient received prompt intravenous antibiotics; however, hepatic abscesses can be easily misinterpreted as cystic hepatic metastases which can delay appropriate therapy. Streptococcus anginosus was found to be responsible for the formation of the liver abscesses visualized on computed tomography (CT) scan. Similar to Streptococcus bovis, knowledge in the literature is arising about the association between $S$. anginosus and gastrointestinal malignancies. This case highlights the importance of identifying concomitant primary GISTs with intra-hepatic abscesses, as these lesions can be easily misconstrued as liver metastases and consequently mismanaged. We herein emphasize that hepatic abscesses are a potential sequela of GISTs and should thus prompt further investigation for potential malignancies, if warranted, so that there is no delay in treatment of these gastrointestinal tumors.
\end{abstract}

Manuscript submitted February 13, 2020, accepted March 12, 2020

aDepartment of Medicine, Jersey Shore University Medical Center, Hackensack Meridian Health, Neptune, NJ, USA

${ }^{b}$ Department of Medicine, Jefferson Health New Jersey, Jefferson University Hospital, Stratford, NJ, USA

${ }^{\mathrm{c} D e p a r t m e n t}$ of Surgery, Jersey Shore University Medical Center, Hackensack Meridian Health, Neptune, NJ, USA

dDepartment of Pathology, Jersey Shore University Medical Center, Hackensack Meridian Health, Neptune, NJ, USA

eUniversity de Santiago de Compostela, Galicia, Spain

${ }^{f}$ Corresponding Author: Gabriella A. Conte, Department of Medicine, Jersey Shore University Medical Center, Hackensack Meridian Health, Neptune, NJ 07753, USA. Email: gabriella.conte@hackensackmeridian.org

doi: https://doi.org/10.14740/wjon1270
Keywords: Gastrointestinal stromal tumors; Small bowel; Liver abscess; Streptococcus anginosus; Imatinib mesylate; Gleevec

\section{Introduction}

Gastrointestinal stromal tumors (GISTs) are the most common type of mesenchymal neoplasm of the gastrointestinal tract [1, 2]. These tumors arise from CD34-positive interstitial cells of Cajal (ICCs) stem cells from the gut wall [1-3]. Diffuse ICC hyperplasia found in the adjacent gut wall of resected tumors has led to the finding that these lesions are precursors to GISTs [4]. These neoplasms primarily affect the stomach $(60 \%)$ and proximal small intestine $(30 \%)$ but can arise in any part of alimentary canal [1]. Occasionally, they metastasize to the omentum, mesentery, peritoneum and liver [1,2]. GISTs are distinguished from other spindle cell tumors of the gastrointestinal tract by their positive CD117 (c-KIT) and DOG1 expression on immunohistochemistry [5]. About $85 \%$ of these tumors carry activating mutations in the c-KIT receptor tyrosine kinase or, less frequently, in platelet-derived growth factor receptor alpha (PDGFR $\alpha$ ) genes $[1,6,7]$. This finding has revolutionized treatment via molecularly targeted therapy with tyrosine kinase inhibitors.

Despite being the most common non-epithelial tumor of the gastrointestinal tract, GISTs represent merely $1.0 \%$ of all primary gastrointestinal neoplasms with an annual incidence of 10 - 14 cases per million people $[8,9]$. GISTs mortality rates differ due to the significant variability in size and malignant behavior of GISTs. The 5-year overall mortality for GIST size $<$ $2 \mathrm{~cm}$ is $30.9 \%$ compared to $35 \%$ for all GIST sizes [9]. Meanwhile, the 5-year relative survival rate in patients with metastatic GIST is 52\% compared to that of localized GIST (94\%) [10]. These tumors are often seen in middle-aged and elderly patients. The clinical presentation of GISTs varies based on tumor size and location of the primary lesion. Although some patients with GISTs are asymptomatic, many can present with vague symptoms including abdominal pain, early satiety, abdominal distension, or gastrointestinal bleeding. Therefore, for most patients the initial detection of GIST may be an inciden- 
tal finding or result from evaluation of nonspecific symptoms. Few uncommon cases of GIST presenting with tumor-bowel fistula or liver abscesses have been reported in the literature [11-13]. We report a rare case of a patient with multiple liver abscesses associated with a primary small bowel GIST. Radiographically these abscesses could have been misinterpreted as cystic hepatic metastases. We thus highlight the importance of recognizing liver abscesses as potential sequelae of GISTs to facilitate appropriate and timely treatment.

\section{Case Report}

A 66-year-old male patient with a history of gastroesophageal reflux disease presented to the emergency department with a 1-day history of dull, non-radiating right upper quadrant abdominal pain. Associated symptoms included fever, rigors, night sweats, myalgia and decreased appetite for the last 1 week. The patient denied recent weight loss, nausea or vomiting, dysphagia, or change in bowel habits. The patient did not have a significant tobacco or alcohol use history. He reported recent travel to Florida 3 weeks prior. The patient had no known causes of immunosuppression such as HIV and his only medication was omeprazole $40 \mathrm{mg}$ daily. The patient denied any family or personal history of malignancy or genetic disorders. Previously, outpatient esophagogastroduodenoscopy and colonoscopy were unremarkable. His surgical history was significant for laparoscopic repair of bilateral inguinal hernias performed at this facility 4 years prior.

At initial presentation, his vital signs were as follows: blood pressure: 120/68 mm $\mathrm{Hg}$; heart rate: 101 beats per minute; respiratory rate: $18 / \mathrm{min}$; body temperature: $37.8{ }^{\circ} \mathrm{C}$; oxygen saturation: 95\% room air. Physical examination demonstrated mild tenderness in the right upper quadrant and right flank area. The patient did not have lymphadenopathy, masses or hepatosplenomegaly. On initial complete blood cell count, the white blood cell count was $13,000 / \mu \mathrm{L}$ (range: 4,500 $11,000 / \mu \mathrm{L})$. In addition, complete metabolic panel showed an elevated aspartate aminotransferase of $98 \mathrm{IU} / \mathrm{L}$ (range: 10 - 42 IU/L), alanine aminotransferase of 109 IU/L (range: 10 - 60 $\mathrm{IU} / \mathrm{L}$ ), alkaline phosphatase of $219 \mathrm{IU} / \mathrm{L}$ (range: 38 - $126 \mathrm{IU} / \mathrm{L}$ )

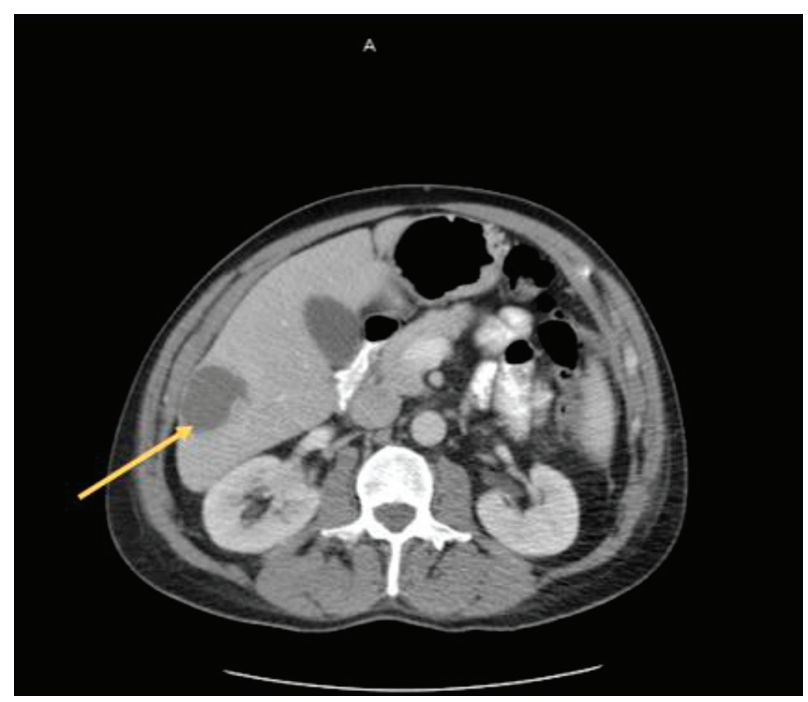

Figure 1. Liver lesion (arrow) identified on axial CT scan of the abdomen and pelvis. CT: computed tomography.

and total bilirubin $2.6 \mathrm{mg} / \mathrm{dL}$ (range: 0.2 - $1.3 \mathrm{mg} / \mathrm{dL}$ ). Hepatitis panel was negative. His erythrocyte sedimentation rate (ESR) and C-reactive protein (CRP) levels were elevated, 34 $\mathrm{mm} / \mathrm{h}$ and $25.98 \mathrm{mg} / \mathrm{dL}$, respectively. Blood and urine cultures were obtained. The patient was sent for right upper quadrant abdominal ultrasound which showed a heterogenous nodular liver with two complex masses in the right lobe, measuring $4.0 \times 4.0 \mathrm{~cm}$ and $3.6 \times 3.6 \mathrm{~cm}$. The patient was started on intravenous (IV) piperacillin/tazobactam for empiric coverage. Subsequent computed tomography (CT) of the abdomen and pelvis with IV contrast revealed multiple low-attenuation lesions involving the entire right hepatic lobe, suspicious for hepatic abscesses versus cystic metastases. The largest lesion involved the inferolateral aspect of the right lobe, measuring $4.2 \mathrm{~cm}$ in greatest diameter (Fig. 1). A notable $4.0 \times 5.7 \mathrm{~cm}$ small bowel mesenteric mass was also identified on imaging (Fig. 2). After discussion with the patient, surgical exploration with liver biopsy was planned.

During exploratory laparotomy, a significant small bowel

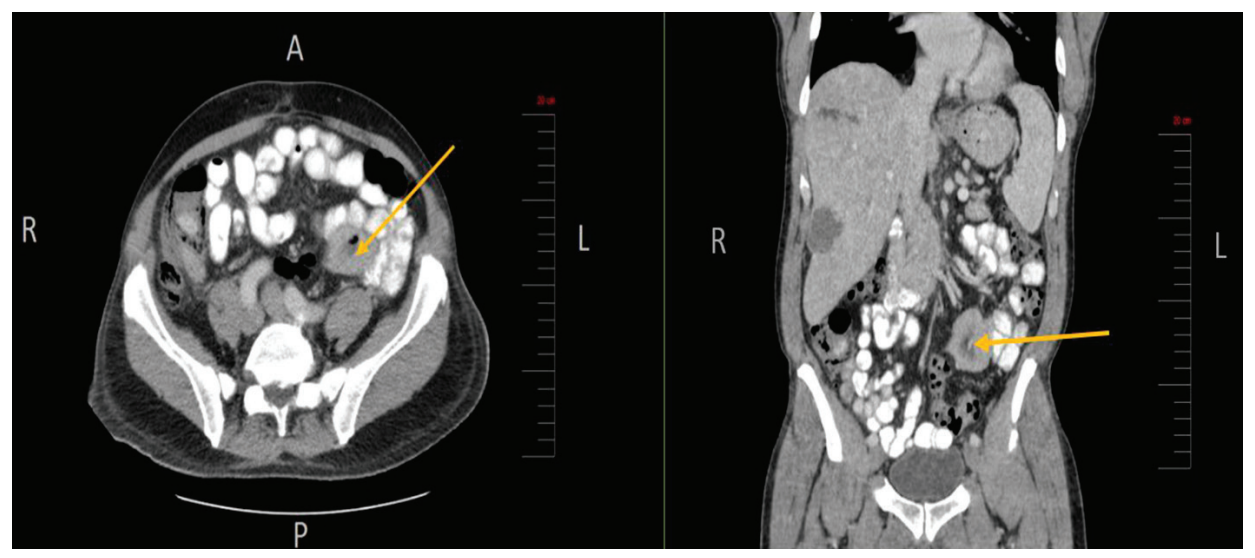

Figure 2. Small bowel mesenteric mass (arrow) identified on CT scan of the abdomen and pelvis (axial and coronal views, respectively). CT: computed tomography. 


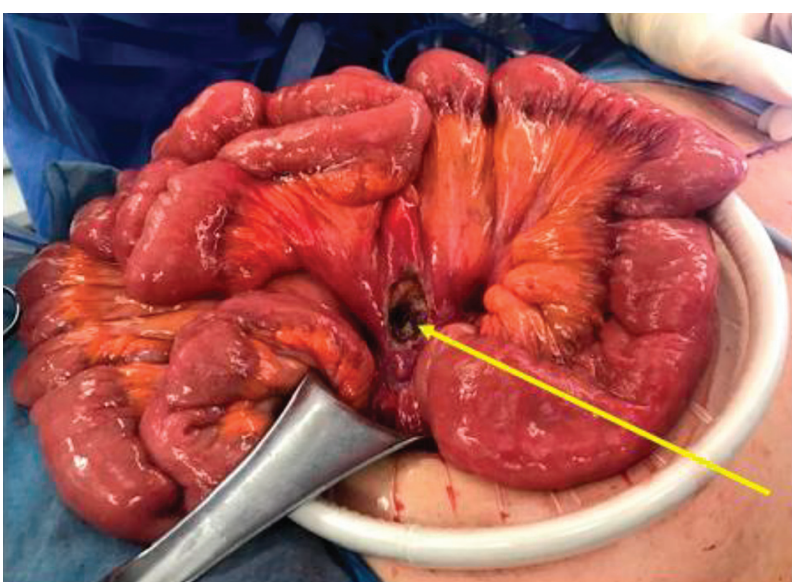

Figure 3. Small bowel mass at the mesenteric root, status post biopsy.

mesenteric mass was found at the root of the mesentery from the first jejunal branches of the superior mesenteric artery extending to the transition point of the proximal ileum (Fig. 3). The mass also involved a portion of the descending colonic mesentery. A small area of the mass was shaved off using electrocautery and sent for frozen section. Preliminary assessment revealed a spindle cell morphology consistent with a GIST. Additional specimen was sent for permanent sample. Given the extensiveness of the mass and likelihood of GIST, the decision was made to refer the patient for neoadjuvant therapy prior to resection. At this junction, an intraoperative liver ultrasound identified the largest abscess. With an 18-gauge needle, $50 \mathrm{~mL}$ of purulent fluid was drained from the lesion and sent for culture. A core needle biopsy of the liver was also obtained to differentiate liver infection versus malignancy. The abdomen was irrigated, and an 8-French pigtail catheter was inserted into the abscess cavity.

On final pathology, the small bowel mesentery mass showed bland appearing spindle cell proliferation with minimal pleomorphism and low mitotic index $(<5 / 50 \mathrm{HPF})$ (Fig. 4a). The tumor cells expressed CD117 (c-KIT) (Fig. 4b) and DOG1, another sensitive and specific marker for GIST, thus confirming the diagnosis of GIST. The tumor cells were also positive for vimentin, smooth muscle actin (SMA), ERG and beta-catenin, and negative for calretinin, CAM5.2, desmin, S-100 and Sox10. Ki67 proliferation index was low $(<3 \%)$. Pathology from the liver lesion showed hepatic parenchyma with extensive necrosis and suppurative inflammation, consistent with abscess. Fluid cultures from the liver were positive for Streptococcus anginosus, for which the patient was treated with IV ampicillin/sulbactam.

The patient had an unremarkable post-operative hospital course. By post-operative day 6 , he had good tolerance of a regular diet, proper bowel functioning, resolution of fevers and normalization of white blood cell count. Subsequent blood cultures remained negative. IV antibiotics were then transitioned to oral amoxicillin/clavulanic acid for an additional 1 week. He was discharged home with a medical oncology referral to establish care and to initiate neoadjuvant therapy with imatinib mesylate (Gleevec) for 6 months, along with a plan thereafter for surgical debulking. Follow-up liver ultrasound post-operatively showed abscess resolution. At 3 months into treatment, the patient has tolerated therapy well and recent CT scan revealed considerable decrease in tumor size, measuring $2.6 \times 2.5 \mathrm{~cm}$.

\section{Discussion}

Approximately $85 \%$ of GISTs harbor oncogenic mutations in one of the receptor protein tyrosine kinases, KIT (CD117) or PDGFR $\alpha$ [1]. Activating mutations in KIT or PDGFR $\alpha$ oncogenes are the key molecular drivers of GIST pathogenesis. These mutations are generally mutually exclusive. The remaining "wild-type" GISTs (10-15\%) do not have detectable KIT or PDGFR $\alpha$ mutations [1]. These GISTs are a heterogenous group which may alternatively have mutations in BRAF, RAS or genes in the succinate dehydrogenase (SDH) complex [1]. The majority of GISTs are sporadic in nature. There are very few known risk factors; however, age over 50 years (mean age at diagnosis 64 years), male gender and certain genetic syndromes have been shown to be predisposing factors for developing GIST [9]. About 5\% of patients with GISTs may have one of several familial autosomal dominant syndromes,

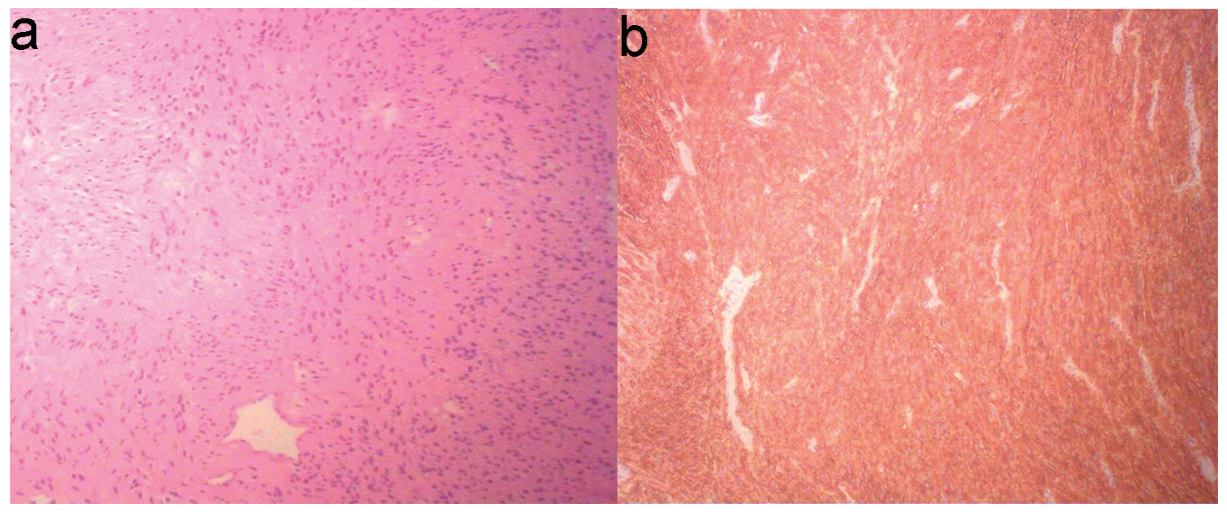

Figure 4. Biopsy of small bowel GIST shows proliferation of bland appearing spindle cells with eosinophilic fibrillar cytoplasm arranging in short fascicular pattern, with minimal pleomorphism and low mitotic index (<5/50 HPF) (a, $\times 10$ objective magnification). The spindle cells are immuno-positive for CD117 (c-KIT) (b). GIST: gastrointestinal stromal tumor. 
including primary familial GIST syndrome, neurofibromatosis type 1 (NF1), or Carney-Stratakis syndrome, which can be associated with heritable mutations in KIT, PDGFR $\alpha$ or SDH genes [14]. Current data suggest that mutational status has prognostic significance for tumor response to therapy.

Understanding that mutational activation of these genes leads to growth of cancer cells has precipitated highly effective targeted molecular therapies. Complete surgical resection remains the mainstay form of therapy for localized primary GISTs, with high 5-year overall survival rates up to $65 \%[15,16]$. However, Gleevec, a selective tyrosine kinase enzyme inhibitor, has become the current standard of therapy for unresectable metastatic or recurrent GIST, demonstrating remarkable tumor response (50-70\%) and prolonged progression-free survival (18 - 20 months) [17-19]. Gleevec is associated with high success rates in both the neo-adjuvant and adjuvant setting. Gleevec is approved as adjuvant therapy for high-risk patients because of its likelihood to eradicate microscopic disease after surgical resection of primary tumor. In a trial conducted by DeMatteo et al, administration of adjuvant Gleevec for 1-year post-resection of localized, primary GIST significantly increased recurrence-free survival compared with placebo (98\% vs. $83 \%$ ) [20]. Gleevec is additionally recommended in the preoperative setting for large or poorly localized primary tumors to reduce tumor size and achieve negative margins with surgical resection [21]. In this case, the small bowel mass at the mesenteric root was unresectable secondary to extensive involvement with the jejunal arteries through the proximal ileum. Thus, neoadjuvant therapy of Gleevec $400 \mathrm{mg}$ daily was initiated.

Our case is of clinical interest due to the rare association of multiple liver abscesses with a primary small bowel GIST. There are few reports in the literature that describe this unique association [22-27]. We herein explore the unique proposed mechanism of pyogenic abscesses in this setting. GISTs can distinctively disrupt the integrity of gastrointestinal mucosa. This in turn creates a pathway for commensal bacteria of the gastrointestinal tract, namely the group Streptococcus milleri, to invade and infect both locally and systemically via portal and systemic circulations [23-25, 28]. This dissemination of organisms can consequently lead to development of liver abscesses. The patient we present was found to have the facultative anaerobic gram-positive organism $S$. anginosus isolated from the liver abscess. S. anginosus, along with Streptococcus intermedius and Streptococcus constellatus, constitutes the group Streptococcus milleri, which inhabits the mucous membranes of the oropharynx, gastrointestinal and genitourinary tracts [23]. There are limited data regarding the association of S. anginosus with gastrointestinal tract malignancies $[23,25$, 27-29]. Our case thus highlights the importance of recognizing this association to facilitate appropriate and timely treatment.

The clinical manifestation of GIST is variable and dependent on tumor site and size; associated imaging findings of an intra-abdominal mass with hepatic lesions can easily be misinterpreted as metastatic disease. Therefore, in this setting, it is critical to consider the concomitance of primary GIST and pyogenic liver abscesses, especially given the significant mortality rate due to liver abscesses (6-26\%) [30]. Early recognition of liver abscesses can expedite appropriate treatment with intravenous antibiotics and percutaneous drainage [13, 27, 28]. Our initial differential of liver abscesses versus cystic metastases prompted immediate IV antibiotic administration as well as surgical exploration with liver biopsy. Core needle liver biopsy results confirmed abscess involvement and treatment with antibiotics was hastily initiated. Therefore, early recognition of liver abscess was important in the management of our patient and for the outcome of his clinical course. This case thus emphasizes the importance of a thorough history, physical examination and review of imaging for patients found to have primary small bowel GIST with infectious involvement.

\section{Conclusion}

We report a rare presentation of a primary small bowel GIST with multiple pyogenic liver abscesses. A 66-year-old patient was found to have a mass at the root of the small bowel mesentery with involvement of the proximal ileum, creating an entry port for translocation of $S$. anginosus into the portal circulation, resulting in hepatic abscesses. This case highlights the importance of identifying concomitant primary GIST with intra-hepatic abscesses, as these lesions can be easily misinterpreted as liver metastases and consequently mismanaged.

\section{Acknowledgments}

The authors gratefully acknowledge the support of the Department of Pathology at Jersey Shore University Medical Center, Hackensack Meridian Health. We thank our patient who kindly gave his consent for this publication.

\section{Financial Disclosure}

This project was not supported by any grants or funding agencies.

\section{Conflict of Interest}

The authors declare that there is no conflict of interest regarding the publication of this paper.

\section{Informed Consent}

The patient described in the case report had given informed consent for the case report to be published.

\section{Author Contributions}

GAC contributed to case selection, planning and drafting the manuscript. JSH contributed to discussion part and edition of the manuscript. RAM contributed to discussion part of the manuscript. DM contributed to case presentation, discussion 
and manuscript revision. XS contributed to the pathological slide review and discussion. EMP contributed to case planning and collecting intra-operative images. FBP contributed to case planning and collecting radiographic images. MJL reviewed and finalized the manuscript. AAC contributed significantly in preparation of the manuscript, editing and final approval.

\section{Data Availability}

The authors declare that data supporting the findings of this study are available within the article.

\section{References}

1. Demetri GD, von Mehren M, Antonescu CR, DeMatteo RP, Ganjoo KN, Maki RG, Pisters PW, et al. NCCN Task Force report: update on the management of patients with gastrointestinal stromal tumors. J Natl Compr Canc Netw. 2010;8(Suppl 2):S1-41; quiz S42-44.

2. Nishida T, Goto O, Raut CP, Yahagi N. Diagnostic and treatment strategy for small gastrointestinal stromal tumors. Cancer. 2016;122(20):3110-3118.

3. Sircar K, Hewlett BR, Huizinga JD, Chorneyko K, Berezin I, Riddell $\mathrm{RH}$. Interstitial cells of Cajal as precursors of gastrointestinal stromal tumors. Am J Surg Pathol. 1999;23(4):377-389.

4. Graadt van Roggen JF, van Velthuysen ML, Hogendoorn PC. The histopathological differential diagnosis of gastrointestinal stromal tumours. J Clin Pathol. 2001;54(2):96102.

5. Hirota S, Isozaki K, Moriyama Y, Hashimoto K, Nishida T, Ishiguro S, Kawano K, et al. Gain-of-function mutations of c-kit in human gastrointestinal stromal tumors. Science. 1998;279(5350):577-580.

6. Heinrich MC, Corless CL, Duensing A, McGreevey L, Chen CJ, Joseph N, Singer S, et al. PDGFRA activating mutations in gastrointestinal stromal tumors. Science. 2003;299(5607):708-710.

7. Hirota S, Ohashi A, Nishida T, Isozaki K, Kinoshita K, Shinomura Y, Kitamura Y. Gain-of-function mutations of platelet-derived growth factor receptor alpha gene in gastrointestinal stromal tumors. Gastroenterology. 2003;125(3):660-667.

8. Tran T, Davila JA, El-Serag HB. The epidemiology of malignant gastrointestinal stromal tumors: an analysis of 1,458 cases from 1992 to 2000. Am J Gastroenterol. 2005;100(1):162-168.

9. Ma GL, Murphy JD, Martinez ME, Sicklick JK. Epidemiology of gastrointestinal stromal tumors in the era of histology codes: results of a population-based study. Cancer Epidemiol Biomarkers Prev. 2015;24(1):298-302.

10. Howlader N, Noone AM, Krapcho M, Miller D, Brest A, Yu M, Ruhl J, et al. SEER cancer statistics review, 19752016, National Cancer Institute, Bethesda, MD. https:// seer.cancer.gov/csr/1975_2016/, based on November 2018 SEER data submission, posted to the SEER website, April 2019.
11. Sorour MA, Kassem MI, Ghazal Ael H, El-Riwini MT, Abu Nasr A. Gastrointestinal stromal tumors (GIST) related emergencies. Int J Surg. 2014;12(4):269-280.

12. Bardell T, Jalink DW, Hurlbut DJ, Mercer CD. Gastrointestinal stromal tumour: varied presentation of a rare disease. Can J Surg. 2006;49(4):286-289.

13. Shamsaeefar A, Hosseini SM, Motazedian N, Razmi T. Enterocolic fistula associated with a gastrointestinal stromal tumor. Indian J Cancer. 2009;46(3):246-247.

14. Wu CE, Tzen CY, Wang SY, Yeh CN. Clinical diagnosis of Gastrointestinal Stromal Tumor (GIST): from the molecular genetic point of view. Cancers (Basel). 2019;11(5):679.

15. Krajinovic K, Germer CT, Agaimy A, Wunsch PH, Isbert C. Outcome after resection of one hundred gastrointestinal stromal tumors. Dig Surg. 2010;27(4):313-319.

16. Nikfarjam M, Kimchi E, Shereef S, Gusani NJ, Jiang Y, Liang J, Sehmbey M, et al. Surgical outcomes of patients with gastrointestinal stromal tumors in the era of targeted drug therapy. J Gastrointest Surg. 2008;12(11):20232031.

17. Blanke CD, Demetri GD, von Mehren M, Heinrich MC, Eisenberg B, Fletcher JA, Corless CL, et al. Long-term results from a randomized phase II trial of standard- versus higher-dose imatinib mesylate for patients with unresectable or metastatic gastrointestinal stromal tumors expressing KIT. J Clin Oncol. 2008;26(4):620-625.

18. Verweij J, Casali PG, Zalcberg J, LeCesne A, Reichardt $\mathrm{P}$, Blay JY, Issels R, et al. Progression-free survival in gastrointestinal stromal tumours with high-dose imatinib: randomised trial. Lancet. 2004;364(9440):1127-1134.

19. Blanke CD, Rankin C, Demetri GD, Ryan CW, von Mehren M, Benjamin RS, Raymond AK, et al. Phase III randomized, intergroup trial assessing imatinib mesylate at two dose levels in patients with unresectable or metastatic gastrointestinal stromal tumors expressing the kit receptor tyrosine kinase: S0033. J Clin Oncol. 2008;26(4):626-632.

20. Dematteo RP, Ballman KV, Antonescu CR, Maki RG, Pisters PW, Demetri GD, Blackstein ME, et al. Adjuvant imatinib mesylate after resection of localised, primary gastrointestinal stromal tumour: a randomised, double-blind, placebo-controlled trial. Lancet. 2009;373(9669):10971104.

21. Lai EC, Lau SH, Lau WY. Current management of gastrointestinal stromal tumors - a comprehensive review. Int J Surg. 2012;10(7):334-340.

22. Kumar A, Artifon EL, Siegel C, Halwan B. Gastrointestinal stromal tumor in association with pyogenic liver abscess. Gastroenterol Hepatol (N Y). 2008;4(11):818-819.

23. Kwon Y, Dang ND, Elmunzer BJ. Gastrointestinal stromal tumor complicated by Streptococcus milleri bacteremia and liver abscess. Eur J Gastroenterol Hepatol. 2009;21(7):824-826.

24. Kim BH, Lee JH, Baik du S, Yun SW, Kim JH, Kong JH, Kim SB. [A case of malignant gastrointestinal stromal tumor of ileum with liver abscess]. Korean J Gastroenterol. 2007;50(6):393-397.

25. Kurtz LE, Greenberg RE. Pyogenic liver abscess associated with a gastrointestinal stromal tumor of the stomach. 
Am J Gastroenterol. 2010;105(1):232-233.

26. Ogura S, Yura M, Sakamoto T, Watanabe Y, Tani N, Yamakita T, Yamazaki M, et al. [A case report of GIST of the small intestine with multiple liver abscesses]. Gan To Kagaku Ryoho. 2011;38(12):2375-2377.

27. Johannsen EC, Sifri CD, Madoff LC. Pyogenic liver abscesses. Infect Dis Clin North Am. 2000;14(3):547-563, vii.

28. Bonenfant F, Rousseau E, Farand P. Streptococcus anginosus pyogenic liver abscess following a screening colon- oscopy. Can J Infect Dis Med Microbiol. 2013;24(2):e4546.

29. Masood U, Sharma A, Lowe D, Khan R, Manocha D. Colorectal cancer associated with streptococcus anginosus bacteremia and liver abscesses. Case Rep Gastroenterol. 2016;10(3):769-774.

30. Chen SC, Huang CC, Tsai SJ, Yen CH, Lin DB, Wang $\mathrm{PH}$, Chen $\mathrm{CC}$, et al. Severity of disease as main predictor for mortality in patients with pyogenic liver abscess. Am J Surg. 2009;198(2):164-172. 